\title{
Comparison of finite-element and homogenization methods for modelling the viscoplastic behaviour of a S2-columnar-ice polycrystal
}

\author{
Jacques Meyssonnier, Armelle Philip \\ Laboratoire de Glaciologie et Géophysique de l'Environnement, CNRS and \\ Université foseph Fourier (UfF-Grenoble I), BP 96, 38402 Saint-Martin d'Hères Cedex, France
}

\begin{abstract}
The main homogenization schemes used to model the behaviour of polycrystalline ice are assessed by studying the particular case of a two-dimensional polycrystal which represents natural S2-columnar ice. The results of the uniform-stress, uniform-strainrate and one-site self-consistent models are compared to finite-element computations. The comparisons were made using the same model of grain, described as a continuous transversely isotropic medium, in the linear and non-linear cases. The uniform-stress and uniform-strain-rate models provide upper and lower bounds for the macroscopic fluidity which are too far from each other to be useful when a degree of anisotropy relevant to ice is considered. Although the self-consistent model gives a weak representation of the interaction between a grain and its surroundings, due to the strong anisotropy of the ice crystal, the resulting macroscopic behaviour is found to be acceptable when compared to the results from finite-element computations.
\end{abstract}

\section{INTRODUCTION}

A number of models have been developed recently to account for the anisotropic behaviour of polar ice and for the evolution of its fabric, in order to improve ice-sheet flow models. Most of these models are based on a "micro-macro" approach which aims at deriving the mechanical properties of a polycrystal of ice (the macroscale) from the knowledge of the behaviour of its constituent grains (microscale). The macroscopic properties are then obtained as averages of the microscopic properties by using a homogenization procedure. Micro-macro models differ from each other by the homogenization methods and the associated hypotheses which are used. Beside this class of models, Morland and Staroszczyk (1998) and Staroszczyk and Morland's (in press) phenomenologically based model incorporates all the mechanisms possibly involved on the grain scale into one internal variable (the total current strain). By construction, this model cannot provide any information on what can be experienced by an individual grain at the microscale, but it should prove to be very efficient to solve large-scale flow of ice sheets. Most of the micro-macro models developed to model the anisotropic behaviour of ice are based on the assumption of a uniform state of stress in the polycrystal (Lliboutry, 1993; Azuma, 1994; Van der Veen and Whillans, 1994; Mangeney and others, 1997; Gödert and Hutter, 1998; Gagliardini and Meyssonnier, 1999), and a few are based on a self-consistent scheme (Castelnau and others, 1996; Meyssonnier and Philip, 1996). Taylor-type models based on the assumption of a uniform strain rate in the polycrystal, and widely used in the metallurgy community, have been dismissed by glaciologists due to the very strong anisotropy of ice.

A comparison of the uniform-stress, uniform-strain-rate, and self-consistent models has been given by Castelnau and others (1996). The aim of the present paper is to assess the results given by these three types of micro-macro models by comparison to finite-element simulations. As a first approach, the macroscopic behaviour of a two-dimensional polycrystal is studied, which makes the numerical computations easier. Nonetheless, this problem is not purely academic since its solution can be applied to natural S2columnar ice loaded in its plane of isotropy. On the other hand, since the general trends observed in a two-dimensional situation are likely to occur in the modelling of a three-dimensional polycrystal, this work is relevant to the problem of modelling the anisotropic behaviour of polar ice.

\section{GRAIN AND S2-IGE CONSTITUTIVE MODELS}

A further simplification, which allows analytical calculations, is made by modelling the ice grain as a continuous incompressible medium. The hexagonal symmetry of ice is taken into account by assuming that the grain is transversely isotropic with its rotational symmetry axis parallel to the ice crystal $c$ axis. When the behaviour is linear, this model is fully equivalent to the description of crystal viscoplasticity by dislocation glide in the basal-, prismatic- and pyramidalslip systems (see the Appendix). In the non-linear case this equivalence is no longer satisfied, however, the transversely isotropic model of grain should be acceptable when basal glide is the dominant mechanism (see the Appendix).

$\mathrm{S} 2$ ice grows from the surface of calm water in a unidirectional temperature gradient. Owing to the process of growth, the grains are elongated columns aligned along the direction of growth, and their $c$ axes are oriented randomly in the plane perpendicular to that direction (see Fig. 1). Therefore 
the polycrystal of S2 ice exhibits macroscopically transverse isotropy.

In the following, the material-symmetry reference frame of a grain is denoted by $\mathcal{R}$, and that of the $\mathrm{S} 2$-ice polycrystal by $\overline{\mathcal{R}}$. The rotational symmetry $x_{3}$ axis of $\mathcal{R}$ is the grain $c$ axis, whereas the $\bar{x}_{3}$ axis of $\overline{\mathcal{R}}$ corresponds to the long direction of the columnar grains. Both grain and polycrystal are incompressible, so their constitutive relation for linear behaviour can be expressed in $\mathcal{R}$ and $\overline{\mathcal{R}}$, respectively, by

$s_{11}-s_{22}=2 \eta_{12}\left(d_{11}-d_{22}\right), \quad s_{33}=2 \eta_{12} \frac{4 \alpha-1}{3} d_{33}$,

$s_{23}=2 \eta_{12} \beta d_{23}, \quad s_{31}=2 \eta_{12} \beta d_{31}, \quad s_{12}=2 \eta_{12} d_{12}$,

where $\mathbf{s}$ is the deviatoric stress tensor and $\mathbf{d}$ denotes the strain-rate tensor (cf. Lliboutry, 1993). The parameters in Relation (1) are defined as follows:

$\eta_{12}$ is the viscosity for shear in the plane of isotropy $\left(x_{1}, x_{2}\right)$,

$\alpha$ is the ratio of the axial viscosity along the $x_{3}$ axis to the axial viscosity in the plane of isotropy $\left(x_{1}, x_{2}\right)$ (i.e. viscosities corresponding to uniaxial compression along these axes),

$\beta$ is the ratio of the viscosity for shear parallel to the plane of isotropy to $\eta_{12}$.

When $\alpha=\beta=1$ the medium is isotropic and Relation (1) reduces to the Newtonian viscous law with $\eta_{12}=\eta$, i.e. $s_{i j}=2 \eta d_{i j}$.

A generalization of (1) to the non-linear behaviour (see Meyssonnier and Philip, 1999) is obtained by replacing $\eta_{12}$ in (1) by an apparent viscosity defined as

$$
\eta^{\star}=A^{-1 / n} \dot{\gamma}_{\diamond}^{(1-n) / n}=A^{-1} \tau_{\diamond}^{1-n},
$$

where

$$
\dot{\gamma}_{\diamond}^{2}=(4 \alpha-1) d_{33}^{2}+\left(d_{11}-d_{22}\right)^{2}+4 d_{12}^{2}+4 \beta\left(d_{23}^{2}+d_{31}^{2}\right),
$$

and

$$
4 \tau_{\diamond}^{2}=\frac{9}{4 \alpha-1} s_{33}^{2}+\left(s_{11}-s_{22}\right)^{2}+4 s_{12}^{2}+\frac{4}{\beta}\left(s_{23}^{2}+s_{31}^{2}\right) .
$$

The two invariants $\dot{\gamma}_{\diamond}$ and $\tau_{\diamond}$ are linked by

$$
\dot{\gamma}_{\diamond}=A \tau_{\diamond}^{n} \text {. }
$$

When the medium is isotropic $\alpha=\beta=1, \dot{\gamma}_{\diamond}=\dot{\gamma}=2 d_{i j} d_{i j}$, $\tau_{\diamond}=\tau=s_{i j} s i j / 2$ and Relation (5) reduces to Glen's law with fluidity parameter $A$.

Relations (1) and (2) are valid for both the grain, with the $x_{3}$ axis corresponding to the grain $c$ axis, and the polycrystal, with the $\bar{x}_{3}$ axis corresponding to the long direction of the columns. In the following, the parameters relative to the polycrystal are overlined.

\section{FINITE-ELEMENT SIMULATIONS}

Owing to the strong anisotropy of the single crystal, the viscoplastic deformation of a grain loaded in a plane which contains its $c$ axis can be considered as two-dimensional and this plane strain assumption can be extended to model the flow of S2 ice loaded in its plane of isotropy. The two-dimensional problem was solved in the S2-polycrystal reference frame $\overline{\mathcal{R}}$ with the $\bar{x}_{3}$ axis perpendicular to the plane of the flow.

The finite-element solution was obtained using a mixed
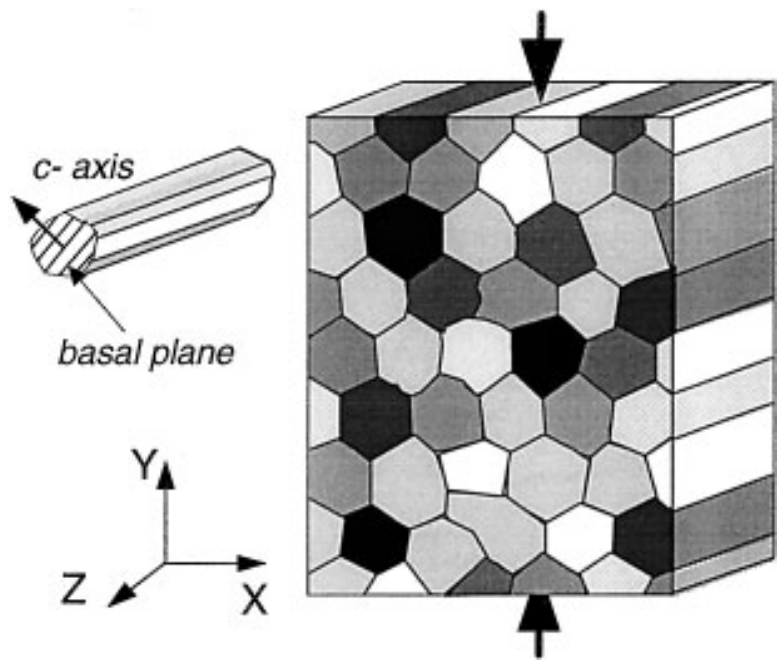

\begin{abstract}
Fig. 1. Structure of S2-columnar ice; the c axis of each columnar grain is perpendicular to its long direction; in the S2-polycrystal, the long axes of the grains are parallel to the same direction, shown as the $\mathrm{Z}$ axis, and the grains $\mathrm{c}$ axes are randomly distributed in the $(X, Y)$ plane (perpendicular to the $\mathrm{Z}$ axis).
\end{abstract}

(velocity-pressure) formulation where the isotropic pressure acts as a Lagrange multiplier to enforce incompressibility (Meyssonnier, 1989). The S2-ice polycrystal was modelled by a regular array of 1024 hexagonal grains with the same dimensions in order to avoid additional stress concentrations due to geometric defects at the triple points. Each grain was modelled by six six-node triangular elements with a quadratic interpolation of the velocities and a linear interpolation of the pressure.

The reference frame of each grain $\mathcal{R}$ was such that its $x_{2}$ and $x_{3}$ axes were in the plane of flow, the $x_{2}$ axis, parallel to the basal plane, being at angle $\varphi$ with respect to the $\bar{x}_{1}$ axis of $\overline{\mathcal{R}}$. The basal-plane orientation of each hexagonal grain was fixed by assigning the same constant value $\varphi$ to the orientation of its six constituent triangular elements. The grain behaviour was modelled using Relations (1) and (2) with $n=1$ and 3 . The finite-element system was obtained by computing the viscosity matrix corresponding to (1) with the apparent viscosity $\eta_{12}^{\star}$ given by (2) in $\mathcal{R}$ before expressing it in $\overline{\mathcal{R}}$.

The macroscopic parameter $\bar{A}$ in Relation (2) was derived from the total dissipated power computed when simulating a uniaxial compression with the direction of loading in the plane of isotropy of the polycrystal. Two types of conditions were tried. The first corresponded to the simulation of a compression test by prescribing a constant vertical component of the velocity on the two horizontal sides of the polycrystal, with a condition of perfect sliding in the horizontal direction. The second consisted of applying a constant vertical-stress vector on the horizontal sides. A condition of free surface on the two other faces was assumed for both types. It was verified that for each type of condition applied to the same polycrystal, the macroscopic behaviour was unchanged if loading was exerted on the vertical faces instead of the horizontal ones (owing to the condition of plane strain $\bar{d}_{33}=0$, the incompressibility condition gives $\bar{d}_{11}=-\bar{d}_{22}$, and Relation (1) for the polycrystal leads to $\bar{s}_{33}=0$, therefore $\bar{s}_{11}=-\bar{s}_{22}$ ).

The results presented were obtained by fixing the value of $\alpha$ at 1 and varying the value of $\beta$, which characterizes the relative resistance to glide in the basal plane, from $\beta=1$ (iso- 


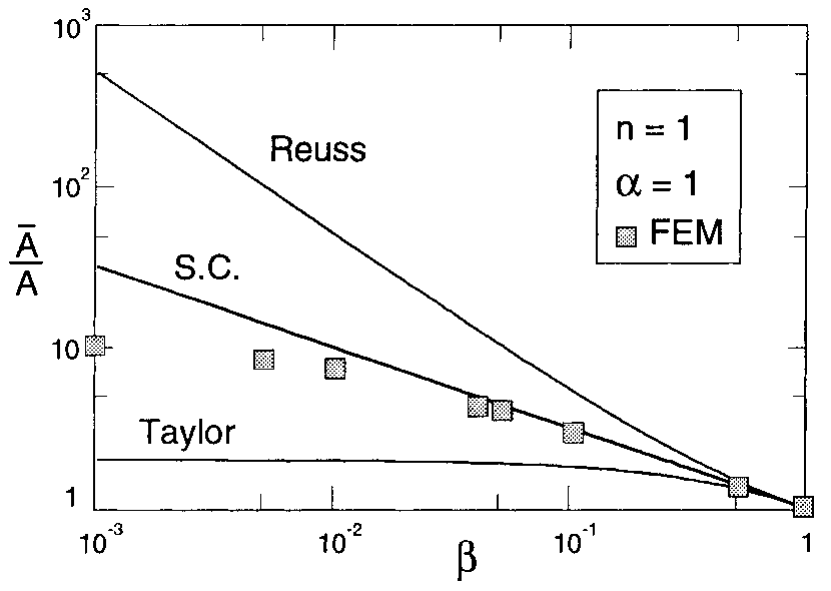

Fig. 2. Evolution of the normalized macroscopic fluidity as a function of $\beta$ given by homogenization models (Reuss: uniform-stress; Taylor: uniform strain rate; S.C: one-site selfconsistent) compared to finite-element results for $n=1$.

tropy) to $\beta=0.001$ which seems to be a lower bound for the single crystal (Duval and others, 1983; Mansuy and others, 1999). In the linear case, five computations were performed for each value of $\beta$ on five polycrystals with different random distributions of the grain orientations. Owing to the large number of grains involved, for a given $\beta$ the values of $\bar{A}$ were not significantly different from a polycrystal to the other (the maximum deviation from the mean value was about $5 \%$ for $\beta=0.001$, decreasing to $1 \%$ for $\beta=0.1$. The influence of the type of applied boundary condition was relatively more pronounced, the velocity-type condition leading to lower values of $\bar{A}$ than the stress-type condition (about $20 \%$ lower for $\beta=$ $0.001,15 \%$ for $\beta=0.01,4 \%$ for $\beta=0.1$, and less than $1 \%$ lower for $\beta>0.5$ ). By prescribing the same velocity to the boundaries of the grains with different orientations which are located at the ends of the polycrystal, the velocity-type condition gives results which tend to be closer to those obtained with the uniform-strain-rate model. The stress-type condition is less constraining, because it is expressed as an integral-type boundary condition in the finite-element formulation. As a consequence, only the results obtained by apply-

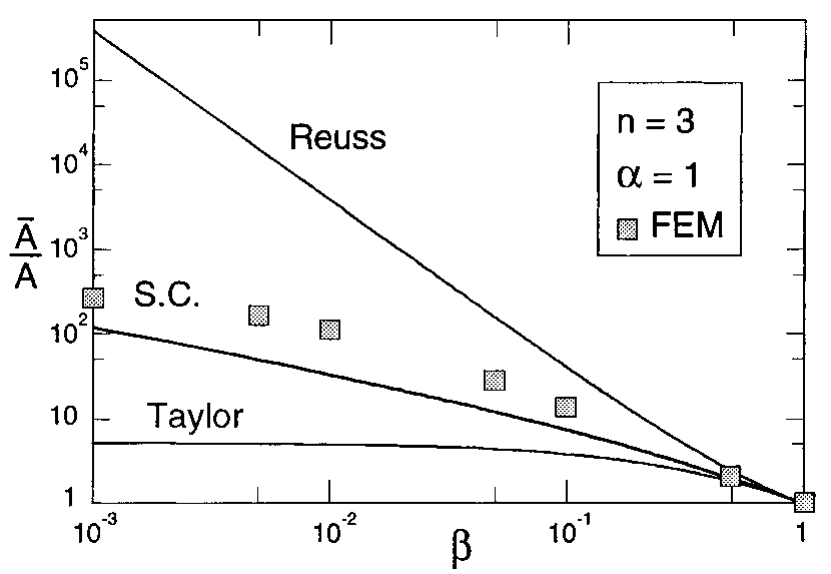

Fig. 3. Evolution of the normalized macroscopic fluidity as a function of $\beta$ given by homogenization models (Reuss: uniform-stress; Taylor: uniform strain rate; S.C: one-site selfconsistent) compared to finite-element results for $n=3$. ing the stress-type condition were considered. Since the number of grains per polycrystal seemed large enough to make the macroscopic viscosity independent of the spatial distribution of the grains, only one polycrystal was considered for the non-linear case with $n=3$. The computed values of $\bar{A}$ are shown in Figures 2 and 3.

\section{HOMOGENIZED BEHAVIOUR}

\section{Notation}

To simplify the expression of the analytical results under the assumption of plane strain, the $\bar{x}_{1}$ and $\bar{x}_{2}$ axes of the polycrystal reference frame $\overline{\mathcal{R}}$ are denoted by $X$ and $Y$ respectively. For a grain, the $x_{2}$ and $x_{3}$ axes of $\mathcal{R}$ are denoted by $x$ and $y$, so that the $c$ axis of the grain is along the $y$ axis, and the basal planes are parallel to the $x$ axis at angle $\varphi$ relative to the $X$ axis of $\overline{\mathcal{R}}$. Loading is exerted in the $(X, Y)=(x, y)$ plane. The components of vectors and tensors are written with lower-case indices when expressed in $\mathcal{R}$ and with upper-case indices when expressed in $\overline{\mathcal{R}}$. Only the quantities related to the polycrystal are overlined. The viscosity in the basal plane of the grain is denoted by $\eta_{z x}=\eta$, whereas the viscosity of the polycrystal in its plane of isotropy $(X, Y)$ is denoted by $\bar{\eta}_{X Y}=\bar{\eta}$. Finally, the mean value of a quantity $Q$ dependent on the grain orientation $\varphi$ is defined by

$$
<Q>=\frac{1}{\pi} \int_{0}^{\pi} Q(\varphi) \mathrm{d} \varphi .
$$

\section{Uniform-stress model}

The uniform-stress model is based on Reuss' assumption that each grain behaves as an isolated crystal subjected to the state of stress applied on the polycrystal boundary. It provides a lower bound for the mean viscosity of the aggregate (Kocks and others, 1998). With the assumption of plane strain applied to the polycrystal, Relation (1) implies $\bar{s}_{Z Z}=\bar{s}_{z z}=0$, and with the assumption of a uniform state of stress, $\mathbf{s}=\overline{\mathbf{s}}$, Relation (1) for the grain becomes

$$
d_{x x}=\frac{1}{2 \eta} \frac{2 \alpha+1}{4 \alpha-1} \bar{s}_{x x}, \quad d_{y y}=\frac{1}{2 \eta} \frac{-3}{4 \alpha-1} \bar{s}_{x x}, \quad d_{x y}=\frac{1}{2 \eta} \frac{1}{\beta} \bar{s}_{x y} .
$$

Note that the $d_{z z}$ component is non-zero. The average viscosity of the polycrystal is obtained by expressing the macroscopic strain rate $\overline{\mathbf{d}}$ as the mean value $<\mathbf{d}>$ of the grains strain rates. Using Relation (7) to express the components of $\mathbf{d}$ in the reference frame $\overline{\mathcal{R}}$ of the polycrystal as functions of $\bar{s}_{X X}$ and $\bar{s}_{X Y}$, the averaging formula (6) leads to

$$
\frac{\eta}{\bar{\eta}}=\frac{\bar{A}}{A}=\frac{1}{2}\left(\frac{\alpha+2}{4 \alpha-1}+\frac{1}{\beta}\right)
$$

in the case of a linear behaviour. For non-linear behaviour, the apparent viscosities given by Relation (2) are expressed in terms of $\tau_{\diamond}$ and $\bar{\tau}_{\diamond}$ given by (4). For $n=3$ the averaging procedure gives, after a simple but lengthy calculation,

$$
\frac{\bar{A}}{A}=\frac{3}{8}\left[\left(\frac{\alpha+2}{4 \alpha-1}\right)^{2}+\frac{2}{3 \beta} \frac{\alpha+2}{4 \alpha-1}+\frac{1}{\beta^{2}}\right] .
$$

The two solutions for $n=1$ and $n=3$ satisfy $\bar{d}_{Z Z}=<d_{Z Z}>=$ 0 . The evolution of $\bar{A}$ as a function of $\beta$ (for $\alpha=1$ ) is shown in Figures 2 and 3 . Note that $\bar{A}$ tends towards infinity as $\beta$ tends towards zero. 


\section{Uniform-strain-rate model}

The Taylor-type models assume a uniform strain rate in the polycrystal. They provide an upper bound for the mean viscosity of the polycrystal (Kocks and others, 1998). With the assumption of plane strain applied to the polycrystal and to the grains, Relation (1) with $\mathbf{d}=\overline{\mathbf{d}}$ becomes

$s_{x x}=2 \eta \frac{2 \alpha+1}{3} \bar{d}_{x x}, \quad s_{y y}=-2 \eta \frac{4 \alpha-1}{3} \bar{d}_{x x}, \quad s_{x y}=2 \eta \beta \bar{d}_{x y}$.

The macroscopic behaviour is obtained by expressing $\overline{\mathbf{s}}$ as the average of s, using (10) expressed in $\overline{\mathcal{R}}$, and (6). In the linear case the calculation is simple and gives

$$
\frac{\eta}{\bar{\eta}}=\frac{\bar{A}}{A}=\frac{2}{\alpha+\beta} .
$$

For $n=3$, the apparent viscosities given by (2) are expressed in terms of $\dot{\gamma}_{\diamond}$ and $\bar{\gamma}_{\diamond}$ given by (3). Since the expression for $\bar{\eta}$ involves a non-integer power of $\dot{\gamma}_{\diamond}$, the results cannot be obtained in an analytical form. The two solutions satisfy $\bar{s}_{Z Z}=<s_{Z Z}>=0$. The evolution of $\bar{A}$ as a function of $\beta$ (for $\alpha=1$ ) is shown in Figures 2 and 3. Note that $\bar{A}$ tends towards a finite value as $\beta$ tends towards zero.

\section{SELF-CONSISTENT ONE-SITE MODEL}

The one-site version of the self-consistent model does not take into account the actual interaction of a grain with its neighbourhood, but considers each grain as an inclusion embedded in a homogeneous equivalent medium (HEM) which has the mean properties of the aggregate. Doing so, all the grains with the same orientation are represented by a unique grain which is assumed to interact with the average of all the possible neighbours of these grains, that is the HEM. The first step consists in solving the inclusion problem to obtain the interaction formula which gives the strain rate in the inclusion as a function of the boundary conditions applied at infinity to the polycrystal. Then the macroscopic properties are derived by homogenization.

For the present application, owing to the assumption of plane strain, the grain is considered as a two-dimensional circular anisotropic inclusion embedded in a two-dimensional isotropic equivalent medium. The detailed solution of the inclusion problem is given by Meyssonnier and Philip (1999). The solution for the points inside the inclusion in the linear case is

$$
d_{x x}=\frac{2}{1+\lambda \alpha} \bar{d}_{x x}, \quad d_{x y}=\frac{2}{1+\lambda \beta} \bar{d}_{x y}
$$

where $\lambda=\eta / \bar{\eta}=\bar{A} / A$ and $\overline{\mathbf{d}}$ is the strain rate applied to the HEM at infinity. Self-consistency is obtained by expressing the macroscopic strain rate $\overline{\mathbf{d}}$ as the mean value of $\mathbf{d}$ (expressed in $\overline{\mathcal{R}}$ ), in which $\bar{\eta}$ is taken as the macroscopic viscosity to be determined. By assuming that all the grains have the same cylindrical shape and are oriented at random, the mean value of $\mathbf{d}$ is given by (6), and the self-consistency equation is obtained as

$$
\frac{1}{1+\lambda \alpha}+\frac{1}{1+\lambda \beta}=1
$$

whose solution is

$$
\lambda=\frac{\eta}{\bar{\eta}}=\frac{\bar{A}}{A}=\frac{1}{\sqrt{\alpha \beta}} .
$$

Note that for a linear behaviour and for inclusions having the same shape, expressing self-consistency on the stresses leads to the same equation. Under the assumption of plane strain, the expression (3) for $\dot{\gamma}_{\diamond}$ is $\dot{\gamma}_{\diamond}^{2}=4\left(\alpha d_{x x}^{2}+\beta d_{x y}^{2}\right)$. With the self-consistent solution (14), the following relation between the grain parameters $\alpha, \beta$ and the viscosity ratio $\lambda$ holds

$$
\frac{\alpha}{(1+\lambda \alpha)^{2}}=\frac{\beta}{(1+\lambda \beta)^{2}}=\frac{\alpha \beta}{(\sqrt{\alpha}+\sqrt{\beta})^{2}},
$$

so that

$$
\dot{\gamma}_{\diamond}^{2}=4 \frac{\alpha \beta}{(\sqrt{\alpha}+\sqrt{\beta})^{2}} \dot{\gamma}_{\diamond}^{2}
$$

Equation (16) shows that the one-site self-consistent solution of the linear problem is such that the power dissipated in a grain does not depend on its orientation.

An estimate of the non-linear behaviour can be obtained by assuming a uniform apparent viscosity in the inclusion and in the HEM (Gilormini, 1996; Kocks and others, 1998). Since $\dot{\gamma}_{\diamond}$ is independent of the grain orientation $\varphi$ for $n=1$, it can be shown that (14) and (16) are still valid for $n=3$. The self-consistent solution (14), written with the expressions for the apparent viscosities of the inclusion and of the HEM given by (2), becomes

$$
\lambda=\frac{\eta^{\star}}{\bar{\eta}^{\star}}=\frac{A^{-1 / n} \dot{\gamma}_{\diamond}^{(1-n) / n}}{\bar{A}^{-1 / n} \dot{\dot{\gamma}}_{\diamond}^{(1-n) / n}}=\frac{1}{\sqrt{\alpha \beta}} .
$$

The macroscopic fluidity parameter $\bar{A}$ is then obtained from (17) and (16) as

$$
\frac{\bar{A}}{A}=\left(\frac{2}{\sqrt{\alpha}+\sqrt{\beta}}\right)^{n-1} \frac{1}{\sqrt{\alpha \beta}} .
$$

\section{DISGUSSION}

Comparisons of the results obtained with the three homogenization methods and with the finite-element computations are shown in Figures 2 and 3 in the form of $\log -\log$ diagrams. The uniform-stress and uniform-strain-rate models provide upper and lower bounds which are too far from each other to be useful. This gap increases as $\beta$ decreases: for $\beta=10^{-3}$ the ratio of the two bounds is about 250 for $n=1$ and about $7.5 \times 10^{4}$ for $n=3$. In the range of $\beta$ relevant for ice, the ratio of $\bar{A}$ given by the self-consistent model (Relation (18)) to the values derived from the finite-element computation is between $1 / 3$ and 3 , depending on the value of $n$. Therefore, as far as the finite-element simulations can be considered, at least in principle, as the best representatives of the actual behaviour of the polycrystal, the self-consistent solution (18) is acceptable.

Inspection of Figures 2 and 3 shows that the parameter $\beta$ of the uniform-stress model can always be tuned to fit a given value of $\bar{A}$ (for this model $\beta$ should not be considered as an intrinsic parameter for the ice-crystal behaviour, but as a model parameter to be adjusted so as to account for the interactions between grains which cannot be accounted for otherwise). This possibility is very limited with the uniformstrain-rate model which gives a maximum value of $\bar{A}$ about 2 for $n=1$, and 5 for $n=3$. The intercomparison of the three homogenization methods done by Castelnau and others (1996) led to the same conclusion.

The self-consistent model is based on the assumption 


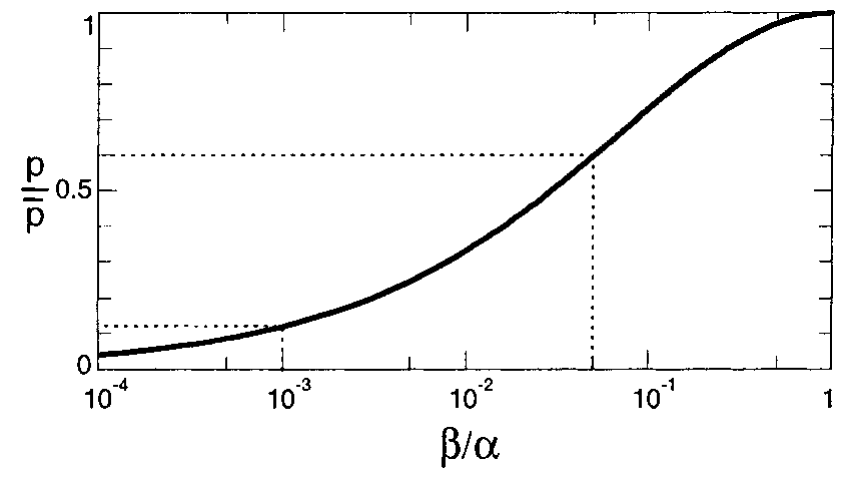

Fig. 4. Ratio of the average of the power dissipated by the grains to the macroscopic dissipated power corresponding to the one-site self-consistent solution (for any $n$ ).

that the neighbourhood of a grain can be considered as a homogeneous medium. For S2 ice, and under the condition of plane strain, this leads to a non-negligible difference between the power dissipated by the macroscopic medium $\overline{\mathcal{P}}$ and the mean power dissipated by the grains $\langle\mathcal{P}\rangle$. Using Relations (5), (16) and (18), $<\mathcal{P}>=<\dot{\gamma}_{\diamond} \tau_{\diamond}>$ is found for any $n$ as

$$
<\mathcal{P}>=4 \frac{\sqrt{\alpha \beta}}{(\sqrt{\alpha}+\sqrt{\beta})^{2}} \overline{\mathcal{P}} .
$$

The ratio $\langle\mathcal{P}\rangle / \overline{\mathcal{P}}$ is less than unity for any value of $\beta / \alpha$ different from 1 , and tends towards zero with $\beta$ (see Fig. 4). It is less than $60 \%$ in the range $0.001 \leq \beta \leq 0.05$ relevant to ice.
This discrepancy is inherent in the one-site model which does not take satisfactorily into account the grain-to-grain interaction. It is all the more important as the grain anisotropy is stronger, and it can explain the results shown in Figures 2 and 3 : the finite-element simulation accounts for the interaction between grains in a better way, and gives a lower macroscopic fluidity than the self-consistent model for $n=1$, whereas, for $n=3$, the stronger stress perturbation obtained with the finite-element simulation leads to a higher fluidity, due to the non-linearity of the constitutive law. Figure 5 is an additional illustration of the interaction between grains obtained by the finite-element method, and seems to indicate that there is no direct correlation between the orientation of a grain and its state of deformation. However, the discrepancy between the finite-element and one-site self-consistent results should be reduced when the polycrystal exhibits a strong fabric with the $c$ axes gathered along the same direction, since the incompatibilities between grains should decrease compared to those arising in an isotropic polycrystal.

\section{GONGLUSION}

The results of the uniform-stress, uniform-strain-rate and self-consistent models have been compared to finite-element computations of the macroscopic behaviour of a two-dimensional polycrystal. The first two models cannot provide useful information on the mean behaviour due to the large difference exhibited in the range of the grain-anisotropy parameter relevant to ice. The macroscopic behaviour predicted by the
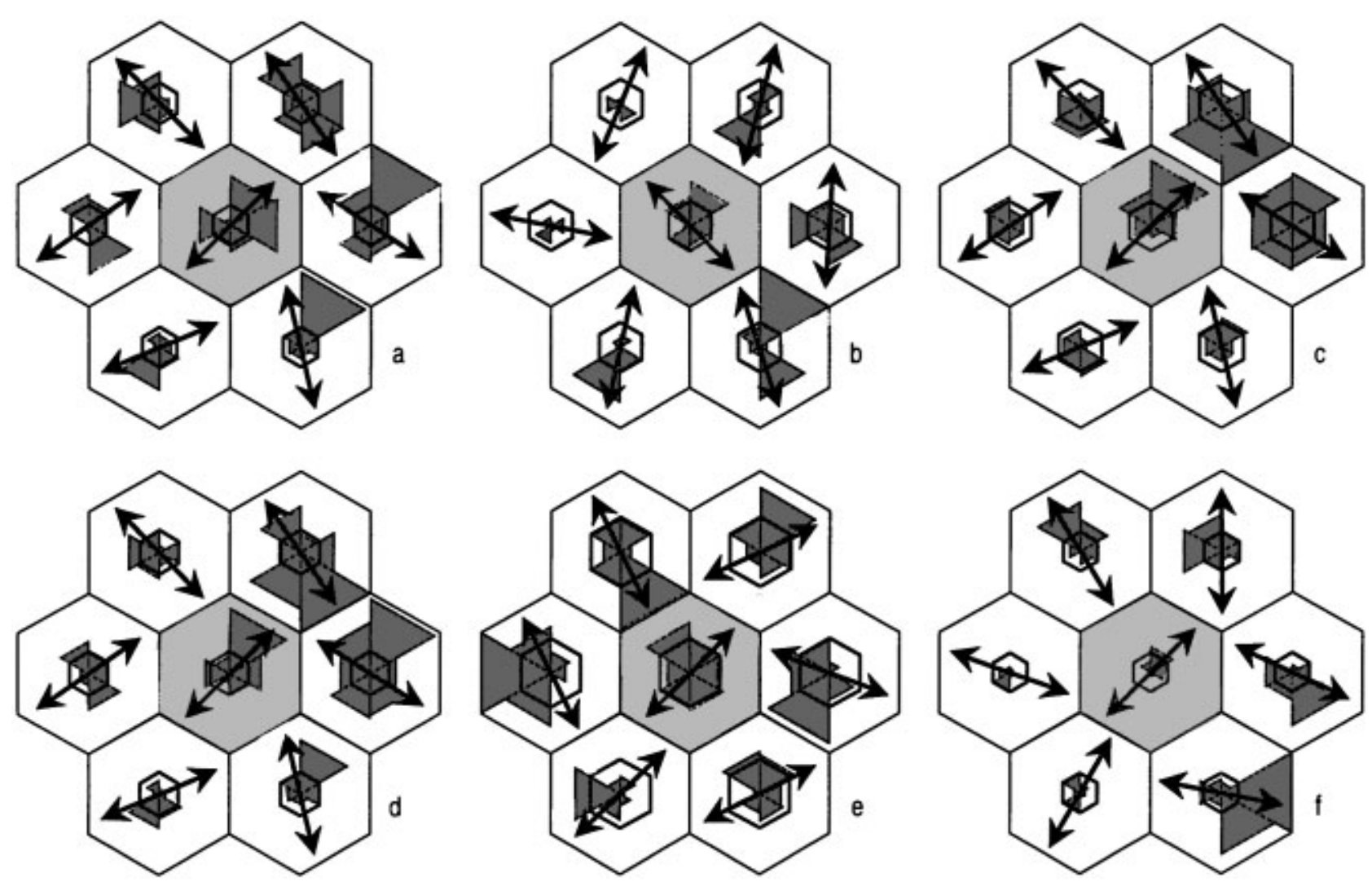

Fig. 5. Power dissipated at the grain scale obtained by finite-element simulations for grains (central hexagon) whose basal planes, shown by the arrows, are at $45^{\circ}$ or $135^{\circ}$ to the direction of compression. The darkened areas show the proportion of power dissipated in a triangular element relative to the maximum $p_{\max }$ dissipated in a triangle in the neighbourhood of the central grain. The inner hexagon shows the mean power dissipated per grain (total power divided by the total number of grains in the finite-element mesh) relative to $p_{\max }$. Different polycrystals $\left(P_{n}\right)$ correspond to different random distribution of the c axes. ( $a$ and $b$ ) $P_{1}, \beta=0.001 ;(c) P_{1}, \beta=0.1 ;(d) P_{1}, \beta=$ 0.01; (e) $P_{3}, \beta=0.01 ;$ (f) $P_{5}, \beta=0.01$. 
one-site self-consistent scheme is acceptable. However it does not take into account the interactions between grains properly and its results should be considered carefully when used to derive local properties at the grain scale.

\section{AGKNOWLEDGEMENTS}

The financial support of the European Commission for the "Fabric Development and Rheology of Polar Anisotropic Ice for Ice Sheet Flow Modelling" project (contract No. ENV4CT95-0125) is gratefully acknowledged. The authors thank D. Dahl-Jensen and R. Staroszczyk for very helpful comments and discussion, and also for corrections to the English.

\section{REFERENGES}

Azuma, N. 1994. A flow law for anisotropic ice and its application to ice sheets. Earth Planet. Sci. Lett., 128(3-4), 601-614.

Castelnau, O., P. Duval, R. Lebensohn and G.R. Canova. 1996. Viscoplastic modeling of texture development in polycrystalline ice with a selfconsistent approach: comparison with bound estimates. 7. Geophys. Res., $\mathbf{1 0 1}$ (B6), 13,851-13,868.

Duval, P., M. F. Ashby and I. Anderman. 1983. Rate-controlling processes in the creep of polycrystalline ice. 7. Phys. Chem., 87 (21), 4066-4074.

Gagliardini, O. andJ. Meyssonnier. 1999. Analytical derivations for the behaviour and fabric evolution of a linear orthotropic ice polycrystal. $\mathcal{F}$. Geophys. Res., 104(B8), 17,797-17,809.

Gilormini, P. 1996. A critical evaluation for various non-linear extensions of the self-consistent model. In Pineau, A. and A. Zaoui, eds. IUTAM Symposium on Micromechanics of Plasticity and Damage of Multiphase Materials. Dordrecht, Kluwer Academic Publishers, 67-74.

Gödert, G. and K. Hutter. 1998. Induced anisotropy in large ice shields: theory and its homogenization. Continuum Mech. Thermodyn., 10(5), 293-318.

Kamb, W. B. 1961. The glide direction in ice. F. Glaciol., 3(30), 1097-1106.

Kocks, U. F., C. N. Tomé and H. R. Wenk. 1998. Texture and anisotropy, preferred orientations in polycrystals and their effect on materials properties. Cambridge, Cambridge University Press.

Lliboutry, L. 1993. Anisotropic, transversely isotropic nonlinear viscosity of rock ice and rheological parameters inferred from homogenization. Int. 7. Plasticity, 9(5), 619-632

Mangeney, A., F. Califano and K. Hutter. 1997. A numerical study of anisotropic, low Reynolds number, free surface flow for ice sheet modeling. F. Geophys. Res., 102 (B10), 22,749-22,764.

Mansuy, P., J. Meyssonnier and A. Philip. 1999. Modelling the ice singlecrystal viscoplastic behaviour. In Hutter, K., Y. Wang and H. Beer, eds. Advances in cold-region thermal engineering and sciences: technological, environmental, and climatological impact. Berlin, etc., Springer-Verlag, 215-224. (Lecture Notes in Physics 533.

Meyssonnier, J. 1989. Ice flow over a bump: experiment and numerical simulations. f. Glaciol., 35(119), 85-97.

Meyssonnier, J. and A. Philip. 1996. A model for the tangent viscous behaviour of anisotropic polar ice. Ann. Glaciol., 23, 253-261.

Meyssonnier, J. and A. Philip. 1999. Remarks on self-consistent modelling of polycrystalline ice. In Hutter, K., Y. Wang and H. Beer, eds. Advances in cold-region thermal engineering and sciences: technological, environmental, and climatological impact. Berlin, etc., Springer-Verlag, 225-236. (Lecture Notes in Physics 533.)

Morland, L. and R. Staroszczyk. 1998. Viscous response of polar ice with evolving fabric. Continuum Mech. Thermodyn., 10(3), 135-152.

Staroszczyk, R. and L.W. Morland. In press. Orthotropic viscous response of polar ice. F. Eng. Math.

Van der Veen, C. J. and I. M. Whillans. 1994. Development of fabric in ice. Cold Reg. Sci. Technol., 22(2), 171-195.

\section{APPENDIX}

A classical approach used in crystal viscoplasticity (Kocks and others, 1998) describes the dependency of the shear strain rate $\dot{\gamma}^{s}$ in the slip system $(s)$ on the resolved shear stress $\tau^{s}$ acting in $(s)$ by a power law of the form $\dot{\gamma}^{s}=$ $\dot{\epsilon}_{0}\left|\tau^{s} / \tau_{\mathrm{c}}^{s}\right|^{n^{s}-1} \tau^{s} / \tau_{\mathrm{c}}^{s}$, where $\dot{\epsilon}_{0}$ is a reference strain rate and $\tau_{\mathrm{c}}^{s}$ characterizes the resistance to shear in $(s)$. Denoting the unit normal to the slip plane by $\mathbf{n}^{s}$ and the unit vector in the slip direction (Burger vector) by $\mathbf{b}^{s}$, expressed in a fixed reference frame, the resolved shear stress on $(s)$ is given by $\tau^{s}=\mathbf{s} \mathbf{m}^{s}$, where $\mathbf{s}$ is the deviatoric stress acting on the crystal, and $\mathbf{m}^{s}$ is the Schmid tensor of components $m_{i j}^{s}=\left(b_{i}^{s} n_{j}^{s}+b_{j}^{s} n_{i}^{s}\right) / 2$. The velocity gradient resulting from slip in $(s)$ is $L_{i j}^{s}=\dot{\gamma}^{s} b_{i}^{s} n_{j}^{s}$ and the corresponding strain rate is $\mathbf{d}^{s}=\dot{\gamma}^{s} \mathbf{m}^{s}$. The strain rate $\mathbf{d}$ resulting from slip on several systems is the sum of $\mathbf{d}^{s}$ over all the active systems: $\mathbf{d}=\sum_{s} \mathbf{d}^{s}$. In the following, we assume that the exponent $n^{s}=n$ in the expression for $\dot{\gamma}^{s}$, is the same for all the active slip systems, and the reference strain rate $\dot{\epsilon}_{0}$ is omitted. Following Castelnau and others (1996), the resistances $\tau_{\mathrm{c}}^{s}$ to shear in the basal-, prismatic- and pyramidal-slip systems are denoted by $\tau_{\mathrm{a}}, \tau_{\mathrm{b}}$ and $\tau_{\mathrm{c}}$, respectively.

The three glide directions in the basal plane $(0,0,0,1)$ are $\langle 2, \overline{1}, \overline{1}, 0\rangle$. The prismatic planes $\{0,1, \overline{1}, 0\}$ provide three slip systems with the same Burger vectors as the basal systems. According to Castelnau and others (1996), the pyramidal planes provide six slip systems in the planes $\{\overline{1}, \overline{1}, 2, \overline{2}\}$, with respective Burger vectors $<\overline{1}, \overline{1}, 2, \overline{3}>$ out of the basal plane.

For $n=1$, from the application of the above formula to ice, the strain-rate components are calculated as

$$
\begin{aligned}
& d_{11}=\frac{3}{8 \tau_{\mathrm{b}}}\left(s_{11}-s_{22}\right)+\frac{3 \mu_{\mathrm{a}}^{2} \mu_{\mathrm{c}}^{2}}{4 \tau_{\mathrm{c}}}\left(s_{11}-s_{22}-6 s_{33}\right), \\
& d_{22}=-\frac{3}{8 \tau_{\mathrm{b}}}\left(s_{11}-s_{22}\right)+\frac{3 \mu_{\mathrm{a}}^{2} \mu_{\mathrm{c}}^{2}}{4 \tau_{\mathrm{c}}}\left(s_{22}-s_{11}-6 s_{33}\right), \\
& d_{33}=\frac{9 \mu_{\mathrm{a}}^{2} \mu_{\mathrm{c}}^{2}}{\tau_{\mathrm{c}}} s_{33}, \\
& d_{23}=\frac{3}{4 \tau_{\mathrm{a}}} s_{23}+\frac{3\left(\mu_{\mathrm{a}}^{2}-\mu_{\mathrm{c}}^{2}\right)^{2}}{2 \tau_{\mathrm{c}}} s_{23}, \\
& d_{31}=\frac{3}{4 \tau_{\mathrm{a}}} s_{31}+\frac{3\left(\mu_{\mathrm{a}}^{2}-\mu_{\mathrm{c}}^{2}\right)^{2}}{2 \tau_{\mathrm{c}}} s_{31}, \\
& d_{12}=\frac{3}{4 \tau_{\mathrm{b}}} s_{12}+\frac{3 \mu_{\mathrm{a}}^{2} \mu_{\mathrm{c}}^{2}}{2 \tau_{\mathrm{c}}} s_{12},
\end{aligned}
$$

where $\mu_{\mathrm{a}}=a / \sqrt{a^{2}+c^{2}}$ and $\mu_{\mathrm{c}}=c / \sqrt{a^{2}+c^{2}}$.

This relation is fully equivalent to the transversely isotropic grain formulation. By identifying Relation (Al) with (1), the relations between the resistances to shear on the slip systems, the ice-lattice parameters $a$ and $c$, and the grain model parameters $\eta_{12}, \alpha, \beta$, are obtained as

$$
\begin{gathered}
\frac{1}{\eta_{12}}=\frac{3}{2}\left(\frac{1}{\tau_{\mathrm{b}}}+2 \frac{\mu_{\mathrm{a}}^{2} \mu_{\mathrm{c}}^{2}}{\tau_{\mathrm{c}}}\right), \quad \alpha=\frac{1}{16 \mu_{\mathrm{a}}^{2} \mu_{\mathrm{c}}^{2}} \frac{\tau_{\mathrm{c}}}{\tau_{\mathrm{b}}}+\frac{3}{8}, \\
\beta=\frac{\tau_{\mathrm{a}}}{\tau_{\mathrm{b}}} \frac{\tau_{\mathrm{c}}+2 \mu_{\mathrm{a}}^{2} \mu_{\mathrm{c}}^{2} \tau_{\mathrm{b}}}{\tau_{\mathrm{c}}+2\left(\mu_{\mathrm{a}}^{2}-\mu_{\mathrm{c}}^{2}\right)^{2} \tau_{\mathrm{a}}}
\end{gathered}
$$

With $c / a=1.629$, the values of $\alpha$ and $\beta$ which correspond to the ratios $\tau_{\mathrm{b}} / \tau_{\mathrm{a}}=\tau_{\mathrm{c}} / \tau_{\mathrm{a}}=70$ used by Castelnau and others (1996) are $\alpha \approx 0.7$ and $\beta \approx 0.02$. For $n=3$, only the components of $\mathbf{d}$ arising from glide on the basal and prismatic systems are found to exhibit rotational symmetry around the $c$ axis (in agreement with Kamb's (1961) results). 